\title{
IMAGEN CORPORAL, CALIDAD DE VIDA Y CONTROL PERCIBIDO EN PACIENTES CON CÁNCER COLORRECTAL: UNA REVISIÓN
}

\author{
Pedro Senabre \\ https://orcid.org/0000-0002-8830-9808 \\ MARIA-CECILIA PHLIPS \\ Universidad Católica de Valencia San Vicente Mártir, Valencia, España
}

Correo electrónico: psperales@gmail.com

Recibido: 25 de octubre del 2021 / Aceptado: 9 de noviembre del 2021

doi: https://doi.org/10.26439/persona2021.n024(2).5570

\begin{abstract}
RESUMEN. El presente artículo tiene como propósito llevar a cabo una revisión acerca de la relación entre el cáncer colorrectal y tres variables psicológicas: la imagen corporal, la calidad de vida y el control percibido. El diagnóstico de una enfermedad crónica es siempre una fuente de estrés que puede desembocar en una pérdida de control, ansiedad, sentimiento de incapacidad y que requiere adaptación por parte del paciente. La revisión teórica se realizó en diferentes bases de datos: EBSCO, PubMed, Medline, Google Académico y libros especializados en este tema. Como conclusión, podemos decir que las dos primeras variables se encuentran estrechamente relacionadas; es decir, personas que padecen este tipo de cáncer presentan peor imagen corporal y una inferior calidad de vida. Finalmente, proponemos que se lleve a cabo una revisión más profunda acerca de este tema, especialmente en la tercera variable: control percibido.
\end{abstract}

Palabras clave: imagen corporal / calidad de vida / control percibido

/ cáncer colorrectal 


\title{
BODY IMAGE, QUALITY OF LIFE AND PERCEIVED CONTROL IN PATIENS WITH COLORECTAL CANCER: A REVIEW
}

\begin{abstract}
In the present article we aimed to realize a systematic review of the literature about the relationship between three subjects (body image, quality of life and perceived control) and patients with colorectal cancer. The diagnosis of a chronic disease is always a source of stress that can lead to loss of control, anxiety, a feeling of incapacity and that requires adaptation on the part of the patient. We used several databases: EBSCO, PubMed, Medline, Google Academic and books specialized in these subjects. We conclude that there is a strong relation between body image, quality of life and colorectal cancer; further research is needed about the connection between perceived control and colorectal cancer.
\end{abstract}

Keywords: body image / quality of life / perceived control / colorectal cancer 


\section{CÁNCER COLORRECTAL}

El cáncer de colon o cáncer colorrectal se origina en el sistema digestivo o sistema gastrointestinal. Se presenta desde el tejido más largo del intestino grueso (colon) hasta el recto (parte final del colon), y comienza con pólipos benignos que lentamente se van convirtiendo en cáncer (Armitage \& Hardcastle, 1984; Bernhard \& Hürny, 1998). De acuerdo con la American Cancer Society (2012), no se conoce exactamente cuál es la etiología del cáncer colorrectal, pero sí se sabe que existen ciertos factores de riesgo que se asocian con esta enfermedad como, por ejemplo, antecedentes familiares, presencia de pólipos colorrectales, enfermedad intestinal inflamatoria, cáncer en otro lugar del cuerpo, ciertos síndromes genéticos, etcétera.

El cáncer colorrectal es una de las causas más frecuentes de muerte relacionadas con cáncer en los Estados Unidos (American Cancer Society, 2012). Concretamente, es la segunda causa tras el cáncer de pulmón. Según los datos del Instituto Nacional de Estadística (como se cita en Morillas et al., 2012), constituye la séptima causa global de fallecimiento en España. En Estados Unidos, las estimaciones de la American Cancer Society en cuanto al cáncer colorrectal para el 2012 indican aproximadamente 103170 casos nuevos de cáncer de colon, 40290 casos nuevos de cáncer de recto y 51690 muertes a causa de esta enfermedad.

Según los datos del Instituto Nacional de Estadística (como se cita en Morillas et al., 2012), en el año 2009 fallecieron en España 14238 personas por este tipo de cáncer, lo que representa alrededor de 260 muertes semanales. Si se compara con los datos publicados el año anterior, esto significa un aumento del 2,93\%. Según Morillas et al. (2012), en España, si no se instauran medidas de detección precoz, se estima que en los próximos años 1 de cada 20 hombres y 1 de cada 30 mujeres presentarán un cáncer colorrectal antes de los 75 años.

En relación con los datos anteriores, la American Cancer Society (2012) estima que la tasa de mortalidad del cáncer colorrectal ha ido disminuyendo en los últimos veinte años, encontrándose un menor número de casos de personas que lo padecen. Gracias a la detección temprana, los pólipos pueden ser identificados y extirpados antes de que se conviertan en cáncer. Sin embargo, Curless et al. (1994) señalan que los síntomas gastrointestinales son muy comunes en la población en general, lo que hace difícil efectuar un diagnóstico temprano. Estos síntomas implican cambios en los hábitos intestinales, sangrado rectal, anorexia, pérdida de peso y dolor abdominal.

Para Fuchs et al. (1994), el riesgo de padecer la misma enfermedad en personas de más de sesenta años con antecedentes familiares es menor en comparación con los más jóvenes, quienes constituyen un grupo de riesgo más alto y vulnerable a padecer este tipo de cáncer y, por tanto, necesitan valoraciones tempranas. En este sentido, Toribara 
y Sleisenger (1995) describen dos estrategias preventivas para el cáncer colorrectal: evaluación y seguimiento. La primera se refiere a evaluar individuos asintomáticos para determinar el nivel de riesgo para el desarrollo de la enfermedad, mientras que la segunda estrategia consiste en el seguimiento de una población con alto riesgo de cáncer colorrectal. Las herramientas de evaluación y de diagnóstico más utilizadas son la colonoscopía, el análisis de sangre oculta en las heces y la sigmoidoscopía.

Una vez que se ha establecido un diagnóstico de cáncer colorrectal, hay varias maneras de proceder según el tipo y la etapa de cada tumor. Las cuatro formas principales de tratamiento son cirugía, radioterapia, quimioterapia y terapias dirigidas (tales como anticuerpos monoclonales). De todas ellas, la cirugía es a menudo el tratamiento más aplicado contra el cáncer en sus etapas iniciales, y es conocida como colectomía o resección segmentaria. Por lo general, se extirpa el cáncer y una parte no afectada del colon a ambos lados del cáncer (así como los ganglios linfáticos cercanos). Posteriormente, los dos extremos del colon se suturan nuevamente. Para el cáncer de colon, usualmente no se necesita una colostomía (abertura en el abdomen para eliminar los desechos del cuerpo, también llamada estoma), aunque algunas veces se puede realizar de manera temporal para aliviar el colon. Por el contrario, en el cáncer rectal, la prevalencia de un estoma permanente es del $10 \%$ al $35 \%$ (Mohler et al., 2008).

Cuando se decide proceder con una colostomía, el paciente tiene dos grandes preocupaciones: por un lado, la amenaza a la vida por el cáncer y, por otro, la integridad corporal afectada por la colostomía y sus consecuencias (Genzdilov et al., 1977). Un cáncer colorrectal suele conllevar problemas físicos, emocionales e interpersonales que influyen tanto en la calidad de vida como en el control percibido y la imagen corporal del paciente.

En el presente trabajo pretendemos analizar con mayor profundidad las tres variables que se han asociado frecuentemente al cáncer colorrectal (imagen corporal, calidad de vida y control percibido). Para ello, definimos cada una de ellas, analizamos las herramientas de evaluación más empleadas y, por último, describimos la relación con el cáncer colorrectal.

\section{IMAGEN CORPORAL}

En 1950, Paul Schilder definió la imagen corporal (IC) en su obra The Image and Appearance of the Human Body (1950) como la imagen de nuestro propio cuerpo que formamos en nuestra mente. Las personas vemos partes de nuestra superficie corporal, tenemos impresiones táctiles, térmicas y dolorosas, pero más allá de estas experiencias inmediatas se encuentra la unidad del cuerpo, que es percibida, pero no como una percepción. Nosotros llamamos esquema corporal a la imagen tridimensional que todo el mundo tiene de sí mismo. Según Slade (1988), la imagen corporal es la representación mental y 
las sensaciones que tenemos del tamaño, la figura y la forma de las distintas zonas del cuerpo o del cuerpo globalmente. Por su parte, para Fisher (1990), la experiencia corporal abarca la percepción y las actitudes hacia la apariencia, el tamaño corporal, la posición corporal, los límites corporales, la competencia corporal y los aspectos relacionados con el estado físico, la salud y la enfermedad, así como el género de uno mismo. De acuerdo con Rosen (1995), la imagen corporal es un concepto que se refiere a la manera en que uno percibe, imagina, siente y actúa respecto a su propio cuerpo. Así, al definir imagen corporal se contempla aspectos perceptivos; aspectos subjetivos, como satisfacción o insatisfacción, preocupación, evaluación cognitiva, ansiedad; y aspectos conductuales.

\section{Evaluación de la imagen corporal}

La imagen corporal es un factor muy importante en el cáncer colorrectal, ya que el protocolo de intervención frecuentemente va asociado a la necesidad de realizar una colostomía, que tiene como resultado un estoma temporal o permanente. Black y Hyde (2004) concluyen que los pacientes con una colostomía perciben una profunda amenaza a su integridad física y su autoconcepto con cambios negativos en su imagen corporal. Según Whistance et al. (2009), otra consecuencia física es, por ejemplo, la pérdida de cabello causada por el tratamiento con quimioterapia. Por esta razón, durante el proceso de evaluación del tratamiento para el cáncer colorrectal, es necesaria una evaluación rigurosa de la imagen corporal de cada paciente.

Uno de los cuestionarios más ampliamente utilizados en población oncológica para evaluar la imagen corporal es la Body Image Scale (BIS) (por ejemplo, Sharpe et al., 2010; Whistance et al., 2009). Es una escala de diez ítems desarrollada para evaluar la IC en pacientes oncológicos. Cada ítem del BIS se responde con una escala de Likert de 0 a 4 , donde 0 es "nada" y 4 es "mucho". Cuanto más alta sea la puntuación obtenida, más grande será la alteración en la imagen corporal.

Otro cuestionario que evalúa la imagen corporal y la dimensión estética después de la cirugía es el Body Image Questionnaire (BIQ) (Scarpa et al., 2009). El BIQ consiste en ocho ítems que evalúan la actitud del paciente hacia su apariencia física (satisfacción con su cuerpo a partir de la operación, la sensación de un cuerpo herido y la sensación de pérdida de belleza como resultado de la cirugía, la dificultad de autoobservación y la sensación de pérdida de características de género). La dimensión estética mide el grado de satisfacción con respecto a la apariencia física de las cicatrices (satisfacción con la cicatriz, descripción de la cicatriz y posición de la cicatriz). Los dos últimos ítems miden autoconfianza antes y después de la cirugía. Cuanto más alta sea la puntuación obtenida, mejor será la imagen corporal.

Otra forma de evaluar la IC es el Draw-A-Person Test de Machover (1949). Consiste en facilitar una hoja en blanco y un lápiz a los pacientes que para que se dibujen a sí mismos. 
No se les proporciona más instrucciones. Cuando finalizan los dibujos, serán evaluados por terapeutas del arte con las siguientes indicaciones: tienen que estimar el grado de obviedad de varios factores con una escala de Likert que va de 1 ("nada obvio") hasta 4 ("muy obvio"). Los factores son omisión de cuerpo, que indica confusión de la identidad propia y de la IC (Abraham, 1989); omisión de ojos, que implica un sentido de depresión, ansiedad e impotencia (Oster \& Montgomery, 1996); líneas desconectadas o inciertas, que revelan ansiedad y falta de control (Yama, 1990); y, por último, si hay presencia de elementos peculiares.

\section{Imagen corporal en cáncer colorrectal}

Anteriormente comentamos que cuando un paciente está diagnosticado con cáncer colorrectal, la intervención más común es la cirugía para eliminar el tumor y, en ocasiones, es necesario realizar una colostomía con el resultado de un estoma - una apertura en el abdomen-, formado durante la colostomía para poder eliminar las heces. Whistance et al. (2009) presentan un estudio sobre la imagen corporal en una muestra de 82 personas con cáncer colorrectal en estadios I hasta IV después de la cirugía. La edad media de los participantes fue 65 años y eran mayoritariamente varones. Los investigadores utilizaron el BIS para evaluar la imagen corporal y observaron que los pacientes con estoma obtuvieron resultados más altos que aquellos sin estoma. Así, los pacientes con estoma son más propensos a tener una alteración en la imagen corporal que aquellos que no tienen estoma.

Gracias a otro estudio realizado por Sharpe et al. (2010), podemos observar diferencias entre pacientes con y sin estoma en relación con la imagen corporal y sus consecuencias psicosociales. En este estudio, recogieron resultados de una muestra de 114 pacientes con diagnóstico de cáncer colorrectal en estadios I hasta IV. Los autores plantearon la hipótesis de que habría mayor alteración en la imagen corporal en pacientes con estoma. Además, la alteración en la imagen corporal sería un predictor de depresión, ansiedad y distrés social. Durante el procedimiento utilizaron el BIS (Hopwood et al., 2001), la Hospital Anxiety and Depression Scale (HADS; Zigmond \& Snaith, 1983) y el Depression Thermometer (DT; National Comprehensive Cancer Network, 2003). En primer lugar, se observó que aquellos pacientes con estoma eran más propensos a presentar una alteración en la imagen corporal y que, a su vez, esta fue una variable mediadora entre el tener un estoma y la depresión, ansiedad y distrés social. Es decir, el estoma no es un predictor directo de la depresión, ansiedad y distrés, sino que la alteración en la imagen corporal que sufren los pacientes con estoma es un mediador entre la presencia de un estoma y la depresión, ansiedad y distrés social.

Lev-Wiesel et al. (2005) realizaron un estudio sobre la IC mediante dibujos creados por 12 pacientes con colostomía planificada, específicamente aquellos con estoma, a 
través del Test de la Figura Humana (Machover, 1949). La muestra consistía en 6 hombres y 6 mujeres con la edad media de 52,7 años. El $86 \%$ estaba casado y tenía una media de 2,5 hijos; por último, el $86 \%$ de los participantes había acabado 12 años de estudios. Los investigadores observaron que después de la colostomía los dibujos eran muy inseguros y había una desconexión de la línea del cuerpo, en comparación con el dibujo de antes de la cirugía. Por otro lado, se podía observar la presencia de elementos peculiares como, por ejemplo, el tumor, la bolsita del estoma o un charco de orina (obsérvese la figura 1) (Lev-Wiesel et al., 2005).

\section{Figura 1}

Dibujos realizados por pacientes con cáncer colorrectal antes y después de la colostomía con estoma

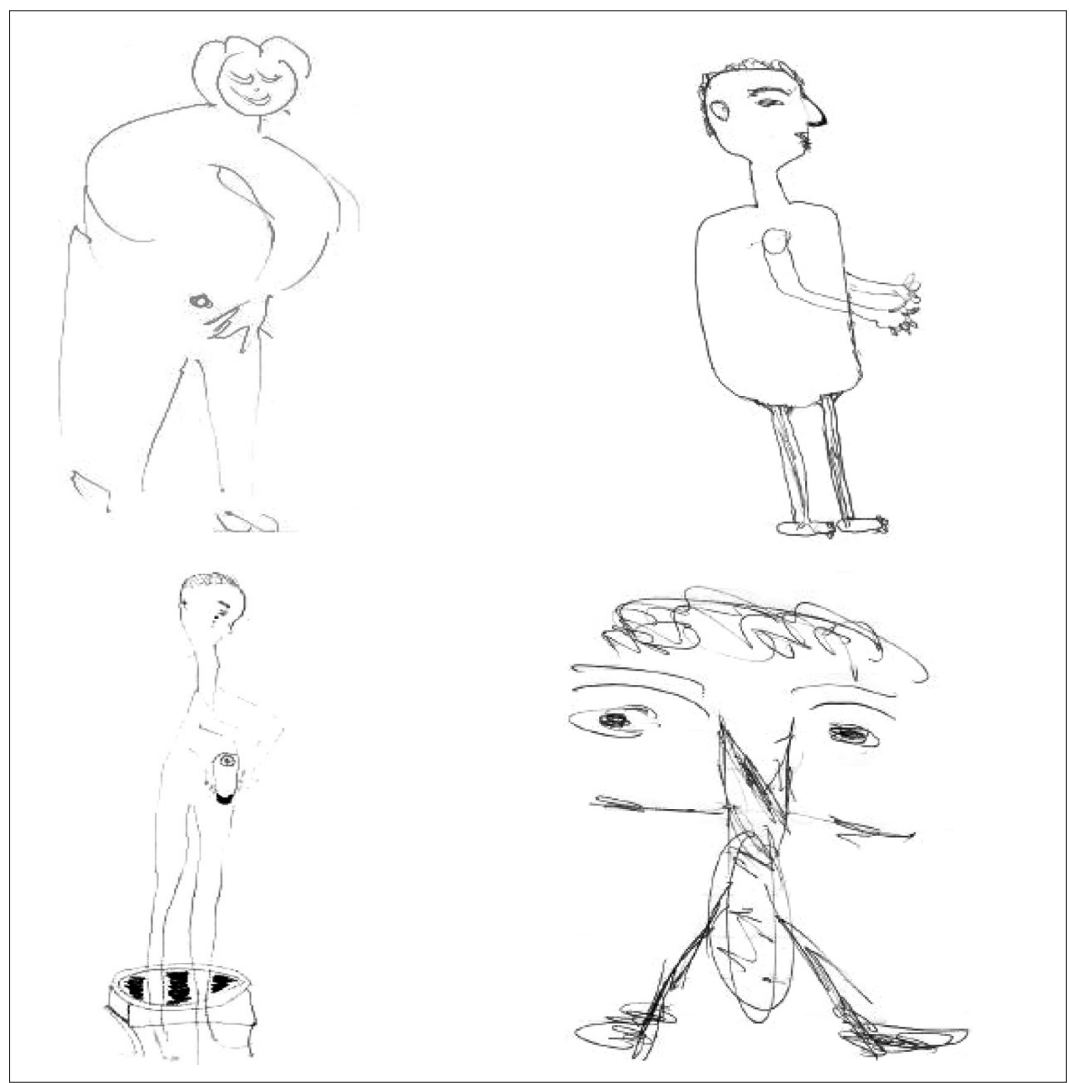

Nota. Izquierda arriba: N, 57 años, casada, madre de tres hijos y con una educación universitaria, se dibujó antes de la colostomía. Izquierda abajo: autodibujo de $\mathrm{N}$, tres meses después de la colostomía. Derecha arriba: Y, 64 años, casado, padre de tres hijos y con una educación universitaria, se dibujó antes de la colostomía. Derecha abajo: autodibujo de Y, tres meses después de la colostomía.

Fuente: Adaptado de Lev-Wiesel et al. (2005) 


\section{CALIDAD DE VIDA}

El concepto calidad de vida es un "término multidimensional de las políticas sociales que significa tener buenas condiciones de vida objetivas y un alto grado de bienestar subjetivo, e incluye la satisfacción colectiva de necesidades a través de políticas sociales" (Palomba, 2002). Según García (2002), la calidad de vida implica la satisfacción de la persona consigo misma, con la familia y los amigos, con su trabajo, con su lugar de residencia y con las actividades que lleva a cabo. Por su parte, Hamming y De Vries (2007) definen calidad de vida como un constructo multidimensional, teniendo en cuenta al menos los aspectos físico y psicológico, y el bienestar social. A esta definición, Hassan y Cima (2007) añaden la importancia de la sexualidad e intimidad como una parte central del bienestar y de la calidad de vida. Según Bloom et al. (2007), una función sexual limitada y una baja satisfacción sexual son factores de riesgo para un grado bajo de calidad de vida.

\section{Evaluación}

Uno de los instrumentos para evaluar la variable calidad de vida es el World Health Organization Quality of Life Instrument, Short-Form (WHOQOL-BREF) (WHOQOL Group, 1998). Evalúa cuatro elementos: factor físico, factor psicológico, relaciones sociales y factor ambiental. Cada ítem se responde con una escala de Likert y se transforman las puntuaciones en una escala de cero a cien. Cuanto más alta es la puntuación, más alto el grado de calidad de vida (Skevington et al., 2004).

El Short-Form Health Survey (SF-36) es otro instrumento para evaluar la calidad de vida en relación con la salud (Apolone \& Mosconi, 1998). Es un cuestionario de 36 ítems que, independientemente del tipo de enfermedad, evalúa los siguientes factores: funcionalidad física, salud física, dolor físico, salud general, vitalidad, funcionalidad social, estatus emocional y salud mental. Cuanto mayor es la puntuación, mayor es la calidad de vida.

El Functional Assessment of Cancer Therapy-Colorectal (FACT-C) se utiliza también para la evaluación de calidad de vida (Ward et al., 1999). Consiste en 35 ítems que describen cinco subescalas: bienestar físico ( 7 ítems), bienestar funcional (7 ítems), bienestar social/familiar (7 ítems), bienestar emocional (7 ítems) y la subescala de cáncer colorrectal (7 ítems). Cuanto mayor sea la puntuación, mejor la calidad de vida.

Por último, el EORTC QLQ-C30 (Aaronson et al., 1993) es un instrumento con 30 ítems divididos en 6 subescalas que miden funcionalidad, 3 subescalas que miden sintomatología y 6 ítems singulares. La subescala que mide funcionalidad engloba la funcionalidad física, emocional, de rol, cognitiva, social y, por último, la funcionalidad global. La subescala que mide la sintomatología evalúa los problemas comunes que los pacientes de cáncer suelen padecer, por ejemplo, el dolor o la fatiga. Se responde a 28 ítems mediante una escala de Likert de 1 a 4, donde 1 es "nada" y 4 "mucho". Los dos 
últimos ítems se responden también mediante una escala de Likert de 1 a 7, donde 1 es "muy pobre" y 7 "excelente".

\section{Calidad de vida en cáncer colorrectal}

Dunn et al. (2006) describieron la influencia del cáncer colorrectal en cinco áreas del bienestar: bienestar psicológico, función física, bienestar social, función laboral/vocacional y función sexual. Evaluaron a los pacientes con diagnóstico de este cáncer en los últimos 18 meses ( $n=66$ ) a través de entrevistas. Con respecto al bienestar psicológico, la mitad de los participantes comentaron no tener ningún cambio. Los que sí reportaron cambios comentaban sufrir depresión y ansiedad sobre el futuro; el miedo a la recaída era una de las preocupaciones principales. Asimismo, la mitad de los pacientes describió la función física con poco o ningún cambio, aunque el estudio destaca que la rehabilitación física se prolongó por espacio de unos meses. Aquellos que percibieron un cambio en la función física señalaron pérdida de fuerza y cansancio como dificultades importantes. Algunos participantes manifestaron que su vida social no sufrió mucho cambio, pero sí destacaron que se sintieron menos cómodos realizando actividades en las cuales era más difícil el acceso a los aseos. Otros comentaron que su vida social se redujo primordialmente por el cansancio. Con respecto a la función laboral, la mayoría de los participantes que todavía trabajaban se reincorporaron después de un tiempo de ausencia. Por último, observaron que el cáncer colorrectal afecta la función sexual en pacientes jóvenes (menores de 60 años), en especial en autoconcepto y relaciones sexuales. Para los participantes masculinos, una preocupación primaria fue la impotencia durante el tratamiento. La mayoría de los pacientes con un estoma temporal anularon totalmente sus relaciones sexuales. Los participantes que todavía no practicaban ninguna relación sexual mostraban la preocupación sobre cómo les podría afectar el cáncer en sus futuras relaciones sexuales.

En una revisión teórica llevada a cabo por Den Oudsten et al. (2012), la función sexual se vio afectada en pacientes con cáncer colorrectal en comparación con la población en general. Según Ross et al. (2007), un estoma es un predictor importante en la disfunción sexual. Destacan que, durante veinticuatro meses después de la cirugía, la disfunción sexual es más alta en los pacientes con estoma que en aquellos sin estoma. Según Jayne et al. (2005), el tipo de cirugía puede predecir el grado de disfunción sexual. Los pacientes con una resección del colon presentan más actividad y deseo sexual a los tres meses después de la cirugía que aquellos con una resección rectal, aunque a partir de los seis meses de cirugía los niveles eran similares. Ameda et al. (2005) observaron que después de cinco años de seguimiento el $64 \%$ de los hombres estaban insatisfechos con su actual funcionalidad sexual.

Hyphantis et al. (2010) realizaron un estudio para analizar la relación entre variables de personalidad, distrés psicológico y calidad de vida en una población con un diagnóstico 
de cáncer colorrectal sin metástasis. Utilizaron una muestra de 144 pacientes con una edad media de 65 años, de los cuales el $67,4 \%$ eran varones. El $70 \%$ tenía el cáncer en el colon y el $25 \%$ en el recto. El 2,1\% de la muestra se encontraba en estadio I, el $59,0 \%$ en estadio II y el $38,9 \%$ en estadio III. Los autores destacan que la calidad de vida de un paciente con cáncer colorrectal alcanza el punto más grave en el momento posoperatorio y empieza a mejorar a partir del tercer mes. Durante los primeros tres años, la calidad de vida es muy baja para los pacientes, mientras que a partir de los tres años la muestra alcanzaba una calidad de vida relativamente uniforme y alta. Del mismo modo, reportaron que la calidad de vida física y mental, y las relaciones sociales disminuyeron moderadamente durante el primer año; por su parte, los síntomas de distrés psicológico (ideas paranoides, psicoticismo, sensibilidad interpersonal, ansiedad y síntomas depresivos) se incrementaron durante el primer año; observaron también que los varones corren más riesgo de desarrollar sintomatología depresiva. El bajo sentido de coherencia era un predictor significativo de depresión, ya que una persona que puntúa alto en sentido de coherencia suele percibir las complicaciones como algo entendible, el tratamiento como algo manejable y la vida con un sentido, aunque las circunstancias puedan cambiar (Wettergren et al., 2004).

La hostilidad fue observada como un predictor de ansiedad, sensibilidad interpersonal y síntomas psicóticos. También observaron que puntuar alto en hostilidad, tener un familiar con cáncer y mostrar síntomas de ansiedad en el momento del diagnóstico puede predecir un aumento de los síntomas de ansiedad durante el primer año. Relacionando las estrategias de afrontamiento con la calidad de vida, observaron que el uso de la negación y el bajo sentido de coherencia predecían una disminución de la calidad de vida mental. Asociaron también variables psicosociales con las relaciones sociales de la calidad de vida. Vieron que el tener un estoma estaba asociado significativamente con una disminución de las relaciones sociales, aunque concluyeron que el distrés psicológico es un predictor más fuerte para las relaciones sociales. Aparte de la presencia de un estoma o no, una baja puntuación del sentido de coherencia es un predictor significativo de pocas relaciones sociales. Hyphantis et al. (2010) encontraron también resultados significativos sobre la represión como predictor de la calidad de vida física. Vos y De Haes (2007) concluyeron que las reacciones emocionales tienden a ampliar síntomas físicos. Esta afirmación nos puede explicar que aquellos pacientes que emplean la represión como estrategia de afrontamiento o aquellos pacientes que se "olvidan" de los estresores, pero a pesar de ello siguen viviendo las consecuencias de sus emociones, muestran peor calidad de vida física (Hyphantis et al., 2010).

En conclusión, según Hyphantis et al. (2010), en una población con cáncer colorrectal sin metástasis, los síntomas de distrés psicológico son más propensos a manifestarse y la calidad de vida disminuye significativamente durante el primer año. Los síntomas de distrés psicológico son predictores de la calidad de vida, junto a las variables de 
personalidad, que también pueden predecir el crecimiento de aquellos síntomas de distrés psicológico y la disminución de la calidad de vida (Yost et al., 2008).

\section{CONTROL PERCIBIDO}

El diagnóstico de una enfermedad crónica es siempre una fuente de estrés que puede desembocar en una pérdida de control, ansiedad, sentimiento de incapacidad. Los factores psicológicos (por ejemplo, personalidad, apoyo social, factores sociodemográficos y cognitivos) tienen un rol importante en la manera de adaptación de cada persona (Neipp et al., 2007). En particular, el control percibido (CP) es uno de los factores psicológicos más importantes que nos puede predecir la forma en que una persona se adapta a una situación de mucho estrés (Helgeson, 1992). Según Lledó (2005), el control percibido alto y la eficacia están relacionados con varios efectos positivos en enfermedades crónicas, incluido un mayor nivel de bienestar, alta motivación, mayor uso de estrategias de afrontamiento y una adaptación personal más positiva.

Existe una gran cantidad de conceptualizaciones y teorías sobre control percibido en la literatura (Skinner, 1995, 1996; Wegner, 2002). Podemos considerar este constructo como multidimensional, ya que su definición engloba diferentes conceptos según distintos autores: la autoeficacia, la autorregulación, la indefensión aprendida, la motivación, etcétera.

La teoría de la conducta planificada (Ajzen, 1991) se centra en el CP sobre la conducta. Define el control percibido como la expectativa de la persona de que su conducta está bajo su control tomando en cuenta la percepción de la facilidad o dificultad de la realización de su conducta.

Desde la teoría de la indefensión aprendida (Abramson et al., 1978), a menor percepción de control, menos previsible es la conducta. Este fenómeno se puede explicar por el hecho de que la persona se muestra "indefensa" ante las demandas del otro, ya que estas son inconsistentes. Esta percepción de incontrolabilidad produce en el sujeto una desmotivación, un déficit cognitivo, por el hecho de que es difícil aprender que sus respuestas son eficaces para controlar determinados resultados $y$, por último, provoca un debilitamiento emocional, ya que se manifiesta una ansiedad seguida de depresión.

La teoría social-cognitiva de Bandura $(1977,1987,1997)$ plantea que el control percibido se puede entender en términos de la autoeficacia, es decir, la habilidad percibida por la persona para la realización de cualquier tarea con el fin de conseguir ciertos resultados concretos. Según el mismo autor, la expectativa de autoeficacia o la eficacia percibida es un determinante importante de la conducta que mejora la salud por la influencia de dos factores. En primer lugar, la autoeficacia puede funcionar como un mediador cognitivo de la respuesta de estrés, ya que aquellos que tienen una alta confianza en su capacidad 
para manejar los estresores a los que se enfrentan reducen las creencias biológicas que pueden dañar la función del sistema inmune y ayudan a que este tenga una adecuada respuesta. En segundo lugar, el grado de motivación que regula el esfuerzo y la persistencia de los comportamientos influye de manera directa sobre el aspecto de si la persona quiere cambiar sus hábitos de salud para unas conductas nuevas y más saludables. En otras palabras, el estilo de vida puede favorecer o perjudicar la salud, lo que capacita a las personas para ejercer cierto control conductual sobre la calidad de su propia salud (Maté et al., 2012).

Como hemos comentado anteriormente que existe una relación entre el control percibido y la motivación, podemos decir que el control percibido guarda una relación muy estrecha con la autorregulación, que hace referencia a la propia motivación o capacidad para iniciar una conducta. Es un concepto integrado en el proceso evolutivo, llamado internalización, que supone la transformación de la motivación extrínseca en motivación intrínseca (García-Martín \& Hombrados-Mendieta, 2002). Esto, a su vez, se puede explicar con el término locus de control interno o externo. El locus de control interno se refiere a la percepción que tiene el sujeto de que los eventos ocurren principalmente como efecto de sus propias acciones, es decir, la percepción de que él mismo controla su vida. Por otro lado, el locus de control externo corresponde a la percepción del sujeto de que los eventos ocurren como resultado del azar, el destino, la suerte o el poder y decisiones de otros. En otras palabras, tales personas dicen que los eventos no se pueden controlar por esfuerzo y dedicación propios, sino que los atribuyen a la responsabilidad de otros (Rotter, 1966).

\section{Evaluación}

A pesar de la poca disponibilidad en la lectura de estudios con una evaluación del CP en pacientes con cáncer colorrectal, nos hemos centrado en estudios que evalúan el CP en la población en general o con cáncer de mama principalmente.

En un estudio llevado a cabo por Beckjord et al. (2009), se elaboró un instrumento multidimensional para evaluar múltiples dimensiones del control percibido en mujeres con un diagnóstico reciente de cáncer de mama. Utilizaron seis factores que, según otros estudios (como Newsom et al., 1996; Taylor et al., 1991; Thompson et al., 1993), representan las diferentes dimensiones del CP: CP sobre los síntomas físicos, emociones, decisiones médicas y de cuidado, consecuencias del cáncer, relaciones con otros y sobre la vida en general. Cada subescala tiene entre dos y seis ítems. Cada uno de ellos se responde con una escala de Likert de 1 ("ningún tipo de control") hasta 4 ("mucho control"). Cuanto mayor la puntuación en cada subescala, mayor el nivel de CP.

En otro estudio longitudinal, Bárez et al. (2008) evaluaron el control percibido en mujeres con cáncer de mama con un instrumento elaborado por los mismos autores, 
llamado Self-Efficacy (SE). Consiste en 36 ítems que describen las preocupaciones más frecuentes de pacientes con cáncer de mama. Obtuvieron estos datos de un estudio realizado por Bárez et al. (2007). Cada ítem se responde, según el nivel de preocupación, con una escala de Likert que va de 0 ("ninguna preocupación") hasta 3 ("extremadamente preocupado"). Al mismo tiempo se les evaluó el nivel de capacidad de superación de aquellas preocupaciones con una escala de Likert de 0 ("ninguna") hasta 3 ("completamente"). En el mismo estudio, utilizaron los autores otro instrumento para evaluar el CP: la Personal Competence Scale (PCS), elaborada originalmente por Wallston (1992). Es una escala de 8 ítems que evalúa las expectativas de cada persona de ser capaz de interactuar de una forma eficaz con su entorno. Para contestar los 8 ítems, se les presenta un rango de 8 hasta 48.

Por su parte, Rowe et al. (2005) realizaron un estudio sobre el locus de control y el riesgo percibido de cáncer de mama con mujeres sanas. Para evaluar el locus de control con respecto a la salud en general, utilizaron la Multidimensional Health Locus of Control Scale (MHLC) (Wallston et al., 1978). Es un cuestionario de 18 ítems que mide 3 dimensiones del LOC (interno, externo y el azar) relacionados con la salud. El MHLC evalúa específicamente la creencia de cada individuo de si su salud general está determinada, sí o no, por su propia conducta. Los 18 ítems se contestan con una escala de Likert de 1 ("totalmente en desacuerdo") hasta 6 ("totalmente de acuerdo"). Cada dimensión consiste en 6 ítems, con lo cual la posible puntuación de cada dimensión varía entre 6 y 36 . Aquella dimensión con la puntuación más alta refleja el LOC de la salud en general de un participante.

Por último, queremos destacar el único estudio encontrado sobre la evaluación de $\mathrm{CP}$ en población con cáncer colorrectal. En aquel estudio utilizaron una entrevista semiestructurada que consistía en preguntas abiertas para que los participantes se animaran a llevar la conversación ellos mismos y para abrir puertas a material para otros posibles estudios. Las preguntas abiertas abarcaban varias dimensiones sobre el CP: “¿Cómo entienden el CP que creen poseer durante el tratamiento?", “Si su CP contribuye a su actitud con respecto al autocuidado, ¿cuánta importancia da al CP en el autocuidado?", “¿Cuánto CP ha sentido durante el tratamiento?”, etcétera. Cada entrevista ocupaba entre treinta a sesenta minutos y fueron grabadas y evaluadas por el investigador (Kidd et al., 2008).

\section{Relación con cáncer colorrectal}

En un primer estudio longitudinal y cualitativo realizado por Kidd et al. (2008), han querido evaluar la idea por parte de los pacientes con cáncer colorrectal del control percibido (CP) en relación con su autocuidado durante la quimioterapia. La muestra $(n=11)$ fue elegida según el criterio de inclusión, que consistía en tener un diagnóstico de CCR y elegir la quimioterapia como tratamiento. La muestra fue mayoritariamente masculina y tenían entre 60 y 70 años. Tres de ellos eran solteros y ocho estaban casados o vivían 
en pareja. Diez de los participantes se situaban en el estadio III de cáncer colorrectal y un paciente se encontraba en el estadio IV. Ya que era un estudio longitudinal, los participantes fueron entrevistados en dos diferentes momentos: la primera entrevista fue antes del inicio del tratamiento y la segunda se realizó seis meses después del tratamiento.

La realización de las entrevistas en dos momentos diferentes les permitió hacer una comparación de posibles cambios con respecto al CP entre el inicio y el final del tratamiento (Kidd et al., 2008). En el mismo estudio han querido observar cómo cada paciente define su concepto de CP. Ellos lo concretaron como su habilidad percibida para limitar, afrontar y manejar activamente, por una parte, el impacto emocional de su enfermedad y, por otra, el impacto físico del tratamiento (Kidd, 2007). Después de las entrevistas semiestructuradas, obtuvieron las siguientes conclusiones. En primer lugar, la percepción del control de los pacientes se mantuvo estable a lo largo del tratamiento. En segundo lugar, aparecieron dos grupos opuestos con respecto al CP: un grupo de personas que tenía un nivel muy alto con respecto al CP durante el tratamiento y un grupo en el cual las personas mostraban un nivel muy bajo con respecto al CP durante el tratamiento.

El grupo con el alto nivel en CP comentaba tener la fuerza para prevenir, controlar y manejar los efectos secundarios del tratamiento. Esto se puede entender gracias a que los pacientes explicaron que su tratamiento tuvo menos impacto sobre su vida diaria de lo que esperaron y experimentaron los efectos secundarios como mínimos. En relación con el autocuidado, aquellos con un alto nivel en CP creían que, con una participación activa, podían manejar las consecuencias secundarias del tratamiento. El rol activo durante el autocuidado era reconocido como importante y necesario para poder mantener el control. De la misma manera comentaron sus maneras de ejercer su control sobre el autocuidado, por ejemplo, juzgando si era el momento para tomar la medicación o para limpiar y curar una herida quirúrgica. Otro hecho importante que lograron sacar a la luz fue que las enfermeras eran cruciales en la enseñanza del autocuidado, pero que después su rol era visto más bien como asistente o de apoyo.

Por su parte, los pacientes que mostraron un bajo nivel en CP no creían tener la habilidad de poder influir en el curso o la naturaleza de los efectos secundarios del tratamiento. Este fenómeno se puede explicar en particular por el impacto debilitante del tratamiento en la salud física y psíquica; experimentaron efectos secundarios muy graves del tratamiento, lo que también puede explicar la postura del bajo nivel en CP. Como resultado de esta experiencia, ellos pensaron que los efectos secundarios se incrementarían y que sería imposible prevenirlos o manejarlos únicamente con su esfuerzo y cuidado. Preferían dejar la responsabilidad del manejo de los efectos relacionados con el tratamiento a los enfermeros y a otros profesionales. Ellos creyeron que era poco necesario un rol activo en el autocuidado por su parte, ya que vieron a los profesionales y los enfermeros como los "expertos" en el cuidado. En otras palabras, aquellos pacientes 
con un bajo nivel en CP suelen adoptar una postura fatalista y pasiva, y evitan cualquier oportunidad o situación que les pueda aumentar el grado de control.

Con estos resultados, podemos decir que la percepción de control de cada paciente está estrechamente relacionada con su percepción de autocuidado con respecto al cuidado de las heridas poscirugía, o a la minimización de los efectos secundarios del tratamiento con quimioterapia. Esta misma percepción nos dice cómo afronta cada paciente los efectos secundarios del tratamiento, ya sea de manera activa o pasiva.

\section{CONCLUSIONES Y DISCUSIÓN}

Tras la revisión de la literatura, podemos señalar con respecto a la variable imagen corporal que existen resultados que indican que aquellos pacientes con estoma son más propensos a mostrar una alteración en la imagen corporal. Queremos recalcar que los estudios revisados se centraron solo en la adaptación psicológica del paciente. Es importante sugerir que hay que prestar también atención a la familia o a la pareja, ya que no solo los pacientes oncológicos se deben adaptar al estoma; también tienen que hacerlo su familia y su pareja. El cambio en la integridad del cuerpo lleva a sentimientos de vergüenza, repugnancia y humillación en el paciente, que a la vez influyen en las relaciones sexuales con su pareja y en las relaciones en general.

Por ello, es importante reconocer la necesidad de apoyo psicológico tanto a los pacientes oncológicos como a los familiares tras la colostomía para que, de esta forma, con la terapia, los pacientes y familiares puedan minimizar los efectos negativos que afectan su bienestar psicológico y social. La terapia podría consistir, en primer lugar, en una atención psicoeducativa sobre las consecuencias de un estoma en la vida social y privada de una persona, tanto al mismo paciente como al familiar. En segundo lugar, sería recomendable trabajar la imagen corporal y el control percibido del paciente para que pueda trabajar la aceptación de su estado y maximizar su autonomía.

En relación con la calidad de vida, podemos recalcar que la población con cáncer colorrectal tiende a presentar con más facilidad síntomas de distrés psicológico y, en consecuencia, la calidad de vida (física, mental y social) disminuye significativamente durante el primer año de enfermedad. Por otro lado, observamos que determinadas variables de personalidad (por ejemplo, sentido de coherencia, hostilidad, negación y represión) también pueden predecir el crecimiento de los síntomas de distrés psicológico y la disminución de la calidad de vida (Hyphantis et al., 2010).

Según los datos encontrados, sería importante recalcar que la evaluación del distrés se debería incluir como herramienta de screening habitual en el caso de pacientes con cáncer colorrectal, ya que el distrés psicológico se incrementa con el tiempo de la enfermedad y predice la calidad de vida. También se tendría que tomar en cuenta la capacidad 
de afrontamiento tanto psicológico como físico de cada paciente para así promocionar su calidad de vida y autonomía. Hyphantis et al. (2011) consideran importante seguir la investigación sobre el impacto de la personalidad, el distrés psicológico y los parámetros de la enfermedad sobre la calidad de vida del paciente, ya que esto nos podría facilitar más información sobre los factores psicológicos que se deberían trabajar en los pacientes con cáncer colorrectal, de manera que se pueda optimizar su calidad de vida.

Según la revisión teórica de Den Oudsten et al. (2012) sobre la disfunción sexual y la calidad de vida en los pacientes con cáncer colorrectal, podemos concluir que estas personas, en comparación con la población en general, son muy propensas a sufrir consecuencias negativas en su calidad de vida por una disfunción sexual. Los mismos autores observaron que los estudios realizados sobre estas variables (calidad de vida y disfunción sexual) recogieron más datos de hombres que de mujeres. Esto podría deberse a que las mujeres suelen ser más resistentes a contestar preguntas sobre la sexualidad (Hassan \& Cima, 2007). Por esta razón, los autores proponen que futuras investigaciones utilicen muestras más amplias y evalúen a pacientes de ambos sexos a fin de extraer conclusiones sobre posibles diferencias entre hombres y mujeres, relacionadas con la calidad de vida y la disfunción sexual en este tipo de cáncer.

Por otro lado, se sabe poco sobre cómo las parejas y los familiares de los pacientes con cáncer colorrectal afrontan la nueva situación con tantos cambios. Y ya que se conoce que la falta de afectividad y una disfunción sexual son los problemas más comunes en un matrimonio con uno de ellos enfermo (Cano et al., 2005), sería interesante realizar una investigación sobre los diferentes tipos de estrategias de afrontamiento que utilizan los familiares y las parejas en esta situación. De esta forma, podríamos dar nuestro apoyo y los recursos necesarios con respecto a este tema en el momento adecuado.

Por último, existe un debate abierto sobre si la disfunción sexual es algo normal o patológico en los pacientes con cáncer colorrectal, ya que la mayoría suelen ser mayores (Camacho \& Reyes-Ortiz, 2005). Un estudio realizado por Den Oudsten et al. (2012) observó una función sexual más baja en pacientes con cáncer colorrectal comparados con la población en general de la misma edad, lo cual podría indicar que este tipo de cáncer influye negativamente en la función sexual, pero futuras investigaciones son necesarias para evaluar con más profundidad la influencia de los factores sociodemográficos como, por ejemplo, la edad y el sexo.

En relación con la variable de control percibido, podemos entender la reciprocidad entre el autocuidado y la percepción del control. Tal y como destacan Kidd et al. (2008), la percepción de control de cada paciente con cáncer colorrectal está estrechamente relacionada con su percepción del cuidado de las heridas poscirugía, o con la minimización de los efectos secundarios del tratamiento con quimioterapia. Cuanto más control percibido tenga el paciente, más afrontará la situación de forma activa y autónoma. Estudiando 
estos datos, el mismo autor propone que las futuras investigaciones se centren en la psicoeducación de los profesionales de la sanidad, en especial, en cómo pueden ayudar a los pacientes a implicarse de forma más activa en su autocuidado para que obtengan una mayor autonomía y un nivel de control más alto sobre su estado actual.

Por otro lado, ya que hay una gran cantidad de definiciones del CP en las diferentes culturas, Kidd et al. (2008) comentan que sería interesante hacer más estudios sobre cómo entienden los pacientes este concepto y que exploren qué aspectos consideran que les harían sentir más control percibido sobre su estado actual. Esta propuesta puede ayudar a los profesionales de la salud a potenciar las estrategias de afrontamiento de cada paciente, incrementar su implicación activa en el autocuidado y mejorar su CP.

A pesar de que este trabajo no pretende ser una revisión exhaustiva, supone un primer intento de revisar los estudios centrados en este tipo de población en oncología, puesto que hasta hoy son escasas las investigaciones encontradas que se centran específicamente en las tres variables analizadas (imagen corporal, calidad de vida y control percibido) en relación con el cáncer colorrectal.

Con respecto a las herramientas de evaluación aplicadas en los estudios, podemos decir que hay una diversidad de instrumentos para evaluar la imagen corporal y la calidad de vida en población oncológica en general, de los cuales cada uno ha sido utilizado para la población específica con cáncer colorrectal. Con respecto a la variable $\mathrm{CP}$, hay que destacar que, a pesar de la poca investigación encontrada al respecto, nos hemos concentrado en los instrumentos para evaluar el CP en la población en general y en la población con cáncer de mama.

Para finalizar esta revisión teórica, queremos proponer que los profesionales de la salud sigan trabajando con pacientes que padecen este tipo de cáncer, pues, como hemos expuesto anteriormente, es escasa la investigación dirigida a esta población. De este modo, con objeto de ayudar a los pacientes y llevar a cabo una intervención de forma adecuada y fructífera, proponemos que los futuros trabajos no solo se enfoquen en el paciente, sino también en el contexto social donde se desenvuelve: su familia, su pareja, etcétera, puesto que el cáncer lo padece la persona enferma y también sus familiares. Es decir, para poder ayudar a una persona de una forma eficaz, es necesario tratar al paciente en todas sus dimensiones (biológica, psicológica, social y espiritual).

Como conclusión, esta revisión pretende abrir nuevas puertas para los profesionales de la psicología, ya que plantea una formación por parte del psicólogo a los especialistas en la salud (enfermeros y médicos) y, por otro lado, se propone una intervención multidisciplinar, es decir que abarque al paciente en todas sus dimensiones. Una intervención centrada en el autocuidado de los pacientes con cáncer colorrectal y en sus familiares mejoraría su calidad de vida. 


\section{REFERENCIAS}

Aaronson, N. K., Ahmedzai, S., Bergman, B., Bullinger, M., Cull, A., Duez, N. J., Filiberti, A., Flechtner, H., Fleishman, S. B., De Haes, J. C., et al. (1993). The European Organization for Research and Treatment of Cancer QLQ-C30: A Quality-of-Life Instrument for Use in International Clinical Trials in Oncology. Journal of National Cancer Institute, 85(5), 365-376.

Abraham, A. (1989). The Exposed and Secret of Human Figure Drawings. Reshafim.

Abramson, L. Y., Seligman, M. E., \& Teasdale, J. D. (1978). Learned Helplessness in Humans: Critique and Reformulation. Journal of Abnormal Psychology, 87(1), 49-74. https://doi.org/10.1037/0021-843X.87.1.49

Ajzen, I. (1991). The Theory of Planned Behavior. Organizational Behavior and Human Decision Processes, 50(2), 179-211.

Ameda, H., Kakizaki, H., Koyanagi, T., Hirakawa, K., Kusumi, T., \& Hosokawa, M. (2005). The Long-Term Voiding Function and Sexual Function after Pelvic NerveSparing Radical Surgery for Rectal Cancer. International Journal of Urology, 12, 256-263. https://doi.org/10.1111/j.1442-2042.2005.01026.x

American Cancer Society. (26 de enero del 2012). ¿Qué es el cáncer de colon y recto? http:// www.cáncer.org/Espanol/cancer/colonyrecto/Resumen/resumen-sobre-elcancer-colonrectal-what-is-key-statistics

Apolone, G., \& Mosconi, P. (1998). The Italian SF-36 Health Survey: Translation, Validation and Norming. Journal of Clinical Epidemiology, 51(11), 1025-1036. https://doi. org/10.1016/s0895-4356(98)00094-8

Armitage, N. C., \& Hardcastle, J. D. (1984). Early Diagnosis of Colorectal Cancer: A Review. Journal of the Royal Society of Medecine, 77(8), 673-676.

Bandura, A. (1977). Self-Efficacy: Toward a Unifying Theory of Behavioural Change. Psychological Review, 83, 191-215.

Bandura, A. (1987). Pensamiento y acción. Martínez Roca.

Bandura, A. (1997). Self-Efficacy: The Exercise of Control. W. H. Freeman and Company.

Bárez, M., Blasco, T., Fernández-Castro, J., \& Viladrich, C. (2007). A Structural Model of the Relationships between Perceived Control and Adaptation to Illness in Women with Breast Cancer. Journal of Psychosocial Oncology, 25(1), 21-43. doi: 10.1300/ J077v25n01-02

Bárez, M., Blasco, T., Fernández-Castro, J., \& Viladrich, C. (2008). Perceived Control and Psychological Distress in Woman with Breast Cancer: A Longitudinal Study. 
Journal of Behavioral Medicine, 32, 187-196. https://doi.org/10.1007/s10865-0089180-5

Beckjord, E. B., Glinder, J., Langrock, A., \& Compas, B. E. (2009). Measuring Multiple Dimensions of Perceived Control in Women with Newly Diagnosis of Breast Cancer. Psychology \& Health, 24(4), 423-438. https://doi.org/10.1080/08870440701832634

Bernhard, J.,\& Hürny, C. (1998). Gastrointestinal Cancer. En J. C. Holland (Ed.), PsychoOncology (pp. 324- 339). Oxford University Press.

Black, P., \& Hyde, C. (2004). Caring for People with a Learning Disability, Colorectal Cancer and Stoma. British Journal of Nursing, 13(16), 970-975.

Bloom, J. R., Petersen, D. M., \& Kang, S. H. (2007). Multidimensional Quality of Life among Long-Term (5+ years) Adult Cancer Survivors. Psycho-Oncology, 16(8), 691-706.

Camacho, M. E., \& Reyes-Ortiz, C. A. (2005). Sexual Dysfunction in the Elderly: Age or Disease? International Journal of Impotence Research, 17(Suppl. 1), s52-s56.

Cano, A., Johansen, A. B., Leonard, M. T., \& Hanawalt, J. D. (2005). What Are the Marital Problems of Patients with Chronic Pain? Current Pain and Headache Reports, 9(2), 96-100.

Curless, R., French, J., Williams, G. V., \& James, O. F. (1994). Comparison of Gastrointestinal Symptoms in Colorectal Carcinoma Patients and Community Controls with respect to Age. Gut, 35(9), 1267-1270. https://doi.org/10.1136/gut.35.9.1267

Den Oudsten, B. L., Traa, M. J., Thong, M. S. Y., Martijn, H., De Hingh, I. H. J. T., Bosscha, K., \& Van de Poll-Franse, L. V. (2012). Higher Prevalence of Sexual Functioning in Colon and Rectal Cancer Survivors Compared with the Normative Population: A Population-Based Study. European Journal of Cancer, 48(17), 3161-3170. https:// doi.org/10.1016/j.ejca.2012.04.004

Dunn, J., Lynch, B., Rinaldis, M., Pakenham, K., McPherson, L., Owen, N., Leggett, B., Newman, B., \& Aitken, J. (2006). Dimensions of Quality of Life and Psychosocial Variables Most Salient to Colorectal Cancer Patients. Psycho-Oncology, 15(1), 20-30.

Fisher, S. (1990). The Evolution of Psychological Concepts about the Body. En T. F. Cash \& T. Pruzinsky (Eds.), Body Images. Development, Deviance, and Change (pp. 3-20). The Guilford Press.

Fuchs, C. S., Giovannucci, E. L., Colditz, G. A., Hunter, D. J., Speizer, F. E., \& Willett, W. C. (1994). A Prospective Study of Family History and the Risk of Colorectal Cancer. The New England Journal of Medicine, 331(25), 1669-1674. https://www.nejm.org/ doi/full/10.1056/NEJM199412223312501 
García, M. A. (2002). Desde el concepto de felicidad al abordaje de las variables implicadas en el bienestar subjetivo: análisis conceptual. Revista Digital, 8(48), 652-660.

García-Martín, M. A., \& Hombrados-Mendieta, M. L. (2002). Control percibido y bienestar subjetivo: un análisis de la literatura gerontológica. Revista Multidisciplinar de Gerontología, 12, 90-100.

Genzdilov, A. V., Alexandrin, G. P., Simonov, N. N., Evtjuhin, A. I., \& Bobrov, U. F. (1977). The Role of Stress Factors in the Postoperative Course of Patients with Rectal Cancer. World Journal of Surgical Oncology, 9(5), 517-523.

Hamming, J. F., \& De Vries, J. (2007). Measuring Quality of Life. British Journal of Surgery, 94(8), 923-924.

Hassan, I., \& Cima, R. R. (2007). Quality of Life after Rectal Resection and Multimodality Therapy. World Journal of Surgical Oncology, 96(88), 684-692.

Helgeson, V. S. (1992). Moderators of the Relation between Perceived Control and Adjustment to Chronic Illness. Journal of Personality and Social Psychology, 63, 656-666.

Hopwood, P., Fletcher, I., Lee, A., \& Al Ghazal, S. (2001). A Body Image Scale for Use with Cancer Patients. European Journal of Cancer, 37(2), 189-197.

Hyphantis, T., Paika, V., Almyroudi, A., Kampletsas, E. O., \& Pavlidis, N. (2010). Personality Variables as Predictors of Early Non-Metastatic Colorrectal Cancer Patients' Psychological Distress and Health-Related Quality of Life: A One-Year Prospective Study. Journal of Psychosomatic Research, 70, 411-421.

Jayne, D. G., Brown, J. M., Thorpe, H., Walker, J., Quirke, P., \& Guillou, P. J. (2005). Bladder and Sexual Function Following Resection for Rectal Cancer in a Randomized Clinical Trial of Laparoscopic versus Open Technique. British Journal of Surgery, 92(9), 1124-1132. https://doi.org/10.1002/bjs.4989

Kidd, L. (2007). An Exploration of Patients' Perceived Control, Self-Efficacy and Involvement in Self Care during Chemotherapy for Colorectal Cancer [Tesis doctoral, Universidad de Stirling]. https://dspace.stir.ac.uk/handle/1893/307\#.YaV5_tDMJnl

Kidd, L., Hubbard, G., O'Carroll, R., \& Kearney, N. (2008). Perceived Control and Involvement in Self Care in Patients with Colorectal Cancer. Journal of Clinical Nursing, 18(16), 2292-2300.

Lev-Wiesel, R., Ziperstein, R., \& Rabau, M. (2005). Using Figure Drawings to Assess Psychological Well-Being among Colorectal Cancer Patients before and after Creation of Intestinal Stomas: A Brief Report. Journal of Loss and Trauma, 10 , 359-367. 
Lledó, A. (2005). La fibromialgia en atención primaria y especializada: determinantes psicosociales del estado de salud percibido y la utilización de recursos sanitarios [Tesis doctoral no publicada]. Universidad Miguel Hernández de Elche, España.

Machover, K. (1949). Personality Projection in the Drawing of the Human Figure. Springfield Publishing.

Mohler, M. J., Coons, S. J., Hornbrook, M. C., Herrington, L. J., Wendel, C. S., Grant, M., \& Krouse, R. S. (2008). The Health-Related Quality of Life in Long-Term Colorectal Cancer Survivors Study: Objectives, Methods, and Patient Sample. Current Medical Research and Opinion, 24(7), 2059-2070.

Morillas, J. D., Castells, A., Oriol, I., Pastor, A., Pérez-Segura, P., Echevarría, J. M., Caballero, B., González-Navarro, A. Bandrés, F., Brullet, E., Iniesta, A., Carballo, F., Bouzas, R., Ariza, A., Ibisate, A., García-Alfonso, P., Escudero, B., Camacho, S., FernándezMarcos, A., ... \& Paz-Ares, L. (2012). The Alliance for the Prevention of Colorectal Cancer in Spain. A Civil Commitment to Society. Journal of Gastroenterology and Hepatology, 35, 109-128. https://doi.org/10.1016/j.gastrohep.2012.01.002

National Comprehensive Cancer Network. (2003). Distress Management: Clinical Practice Guidelines. Journal of the National Comprehensive Cancer Network, 1(3), 344-374.

Neipp, M. C., López-Roig, S., \& Pastor, M. A. (2007). Control Beliefs in Cancer: A Literature Review. Anuario de Psicología, 38(3), 333-335.

Newsom, J. T., Knapp, J. E., \& Schulz, R. (1996). Longitudinal Analysis of Specific Domains of Internal and External Control and Depressive Symptoms in Patients with Recurrent Cancer. Health Psychology, 15(5), 323-331.

Ortego Maté, M. C., López González, S., \& Álvarez Trigueros, M. L. (2012). 14.4.4 Teoría de la autoeficacia de Bandura (Bandura 1977, 1980, 1991, 1997). En Ciencias Psicosociales I. http://ocw.unican.es/ciencias-de-la-salud/ciencias-psicosociales-i/ materiales/bloque-tematico-iv/tema-14.-la-adherencia-al-tratamiento1/14.4.4-teoria-de-la-autoeficacia-de-bandura

Oster, G. D., \& Montgomery, S. S. (1996). Clinical Uses of Drawings. Jason Aronson.

Palomba, R. (24 de julio del 2002). Calidad de vida: conceptos y medidas. Taller sobre calidad de vida y redes de apoyo de las personas adultas mayores [Diapositivas de PowerPoint]. CEPAL. https://docplayer.es/6701163-Calidad-de-vida-conceptos$y$-medidas-rossella-palomba-institute-of-population-research-and-socialpolicies-roma-italia.html

Rosen, J. C. (1995). The Nature of Body Dysmorphic Disorder and Treatment with Cognitive Behavior Therapy. Cognitive and Behavioral Practice, 2(1), 143-166. 
Ross, L., Abild-Nielsen, A. G., Thomsen, B. L., Karlsen, R. B., Boesen, L. H., \& Johansen C. (2007). Quality of Life of Danish Colorectal Cancer Patients with and without a Stoma. Supportive Care in Cancer, 15(5), 505-513. https://doi.org/10.1007/s00520006-0177-8

Rotter, J. B. (1966). Generalized Expectancies for Internal versus External Control of Reinforcement. Psychological Monographs: General and Applied, 80(1), 1-28.

Rowe, J. L., Montgomery, G. H., Duberstein, P. R., \& Bovbjerg, D. H. (2005). Health Locus of Control and Perceived Risk for Breast Cancer in Healthy Women. Behavioral Medicine, 31(1), 33-40. https://doi.org/10.3200/BMED.31.1.33-42

Scarpa, M., Erroi, F., Ruffolo, C., Mollica, E., Polese, L., Pozza, G., Norberto, L., D'Amico, D. F., \& Angriman, I. (2009). Minimally Invasive Surgery for Colorectal Cancer: Quality of Life, Body Image, Cosmesis, and Functional Results. Surgical Encoscopy, 23, 577-582. https://doi.org/10.1007/s00464-008-9884-1

Schilder, P. (1950). The Image and Appearance of the Human Body. International Universities Press.

Sharpe, L., Patel, D., \& Clarke, S. (2010). The Relationship between Body Image Disturbance and Distress in Colorectal Cancer Patients with and without Stomas. Journal of Psychosomatic Research, 70(5), 395-402.

Skevington, S. M., Lofty, M., \& O'Connell, K. A. (2004). The World Health Organization's WHOQOL-BREF Quality of Life Assessment: Psychometric Properties and Results of the International Field Trial. A Report from the WHOQOL Group. Quality of Life Research, 13(2), 299-310.

Skinner, E. A. (1995). Perceived Control, Motivation and Coping. Sage Publications.

Skinner, E. A. (1996). A Guide to Constructs of Control. Journal of Personality and Social Psychology, 71(3), 549-570.

Slade, P. D. (1988). Body Image in Anorexia Nervosa. The British Journal of Psychiatry: The Psychopathology of Body Image, 153(S2), 20-22. https://doi.org/10.1192/ S0007125000298930

Taylor, S. E., Helgeson, V. S., Reed, G. M., \& Skokan, L. A. (1991). Self-Generated Feelings of Control and Adjustment to Physical Illness. Journal of Social Issues, 47(4), 91-109.

Thompson, S. C., Sobolew-Shubin, A., Galbraith, M. E., Schwankovsky, L., \& Cruzen, D. (1993). Maintaining Perceptions of Control: Finding Perceived Control in Low-Control Circumstances. Journal of Personality and Social Psychology, 64(2), 293-304. 
Toribara, N. W., \& Sleisenger, M. H. (1995). Screening for Colorectal Cancer. The New England Journal of Medicine, 332(25), 861-867. https://doi.org/10.1056/ NEJM199503303321306

Vos, M. S., \& De Haes, J. C. (2007). Denial in Cancer Patients, an Explorative Review. Psycho-Oncology, 16(1), 12-25. https://doi.org/10.1002/pon.1051

Wallston, K. A. (1992). Hocus-Pocus, the Focus Isn't Strictly on Locus. Rotter's Social Learning Theory Modified for Health. Cognitive Therapy and Research, 16(2), 183-199. doi: 10.1007/BF01173488

Wallston, K. A., Wallston, B. S., \& DeVellis, R. (1978). Development of the Multidimensional Health Locus of Control (MHLC) Scale. Health Education, 6(2), 161-170.

Ward, W. L., Hahn, W. A., Mo, F., Hernandez, L., Tulsky, D. S., \& Cella, D. (1999). Reliability and Validity of the Functional Assessment of Cancer Therapy-Colorectal (FACT-C) Quality of Life Instrument. Quality of Life Research, 8(3), 181-195.

Wegner, D. M. (2002). The Illusion of Control. Bradford Books.

Wettergren, L., Björkholm, M., Axdorph, U., \& Langius-Eklöf, A. (2004). Determinants of Health-Related Quality of Life in Long-Term Survivors of Hodgkin's Lymphoma. Quality of Life Research, 13, 1369-79. https://doi.org/10.1023/ B:QURE.0000040790.43372.69

Whistance, R. N., Gilbert, R., Fayers, P., Longman, R. J., Pullyblank, A., Thomas, M., \& Blazeby, J. M. (2009). Assessment of Body Image in Patients Undergoing Surgery for Colorectal Cancer. International Journal of Colorectal Disease, 25(3), 369-374.

WHOQOL Group. (1998). Development of the World Health Organization WHOQOL-BREF Quality of Life Assessment. Psychological Medicine, 28(3), 551-558.

Yama, M. F. (1990). The Usefulness of Human Figure Drawings as an Index of Overall Adjustment. Journal of Psychological Assessment, 54(1-2), 78-86.

Yost, K. J., Hahn, E. A., Zaslavsky, A. M., Ayanian, J. Z., \& West, D. W. (2008). Predictors of Health-Related Quality of Life in Patients with Colorectal Cancer. Health and Quality of Life Outcomes, 6, 66.

Zigmond, A. S., \& Snaith, R. P. (1983). The Hospital Anxiety and Depression Scale. Acta Psychiatrica Scandinavica, 67(6), 361-370. 